\title{
Development of Personal Weather Report for Home Security
}

\author{
Richa Gupta and Dhruv Garg \\ Department of Electronics \& Communication, Jaypee Institute of Information Technology, Noida, INDIA
}

\begin{abstract}
With the fast growing technology, it is a great need to get to know about our surrounding weather parameters (temperature, humidity, atmospheric pressure, gas and light intensity) for better development of living community. A complete weather report can easily access the rarest and the farthest information at one's own fingertips. It is based on IoT (Internet of Things), which is an emerging field in which all the devices are connected to a channel and collect the data to gather a complete information through a personnel devices. It is generally used to view the weather parameters with the help of unique API key for a particular user. Wi-Fi module, temperature, pressure, humidity, gas, and light intensity sensors are interfaced with the Arduino. The user is prompted to provide the API key of channel. ESP8266-01 reads the key and sends it to the Arduino. If the key is matched, then the data transmission can be carried out between the channel and the microcontroller. The module is connected to the Wi-Fi through some AT Commands.
\end{abstract}

Keywords: API keys, AT commands, IoT

\section{Introduction}

IoT is simply the network of interconnected things/devices which are embedded with sensors, software, network connectivity and necessary electronics that enables them to collect and exchange data making them responsive [1-3]. More than a concept Internet of Things is essentially an architectural framework which allows integration and data exchange between the physical world and computer systems over existing network infrastructure [4]. When IoT is augmented with sensors and actuators, the technology becomes an instance of the more general class of cyber- physical systems, which also encompasses technologies such as smart grids, smart homes, intelligent transportation and smart cities. Each thing is uniquely identifiable through its embedded computing system but is able to interoperate within the existing internet infrastructure [5]. The devices collect useful data with the help of various existing technologies and then autonomously flow the data between other devices [6]. Current project and such other projects like smart homes such as control and automation of lighting, heating, ventilation, air conditioning etc. uses Wi-Fi for remote monitoring. The present project is very beneficial to modern society aided with the constantly increasing use of internet. It would also work in taking care of home as we could monitor the weather parameters under the Wi-Fi system.

\section{General Description}

The ESP8266 Wi-Fi module is a self-contained SOC with integrated TCP/IP protocol stack that can give any microcontroller access to your Wi-Fi network. The ESP8266 is capable of either hosting an application or offloading all Wi-Fi networking functions from another application processor. ESP8266 module is preprogrammed with an AT command set firmware [5].This module has a powerful enough on- board processing and storage capability that allows it to be integrated with the sensors and other application specific devices through its GPIOs with minimal development up-front and minimal loading during runtime. It contains a selfcalibrated RF allowing it to work under all operating conditions, and requires no external RF parts.

\section{Project Work}

In view of the daily chores of a household the monitoring of real time parameters is highly beneficial for the sustenance of an organized and well informed standard of living. The parameters we are dealing here are LPG (usually used in household for cooking purposes in kitchens through cylinders or pipelines), room temperatures, pressure, humidity present and light sensitivity required for an ambient living. The respective sensors are being utilized for acquisition of the required data to be monitored as shown in Figure 1, 2, 3.BMP180 sensor has been used to monitor the room temperature and pressure and it is soldered onto a PCB with a $3.3 \mathrm{~V}$ regulator, I2C level shifter and pull-up resistors on the I2C pins. It is a 4 pins sensor of which 2 pins uses I2C protocol (SCL and SDA) which are connected to the respective I2C protocol pins on the Arduino i.e. A5 \& A4. It is based on the piezo resistive technology. MQ-2 sensor is a gas sensor which can detect the presence of combustible gases such as i-butane, LPG, hydrogen \& methane. The ionized constituents are detected by the sensing element, which creates a potential difference thus giving output in the form of current. The concentration of the gas detected is then send to the Arduino. 
DHT11 sensor is used for measuring surrounding humidity and temperature. It sends a data of 40 bits both for the temperature and humidity which also includes the checksum byte (bit error check). It operates at a voltage of $+5 \mathrm{v}$ and gives the digital output connected to any of the digital pin of the microcontroller.LDR (Light Detecting Resistance) sensor is used for measuring the light intensity. It is a 3 pin sensor, which requires an input of $+5 \mathrm{v}$ and gives the analog output connected to any of the analog pin of the microcontroller.ESP8266 module is a Wi-Fi module, which is the backbone of this project. This module has inbuilt set of Attention Commands which are required to configure the module. Firstly we flash the ESP8266 module using the software then using the Attention Commands it is set in the Wi-Fi mode [9]. Arduino UNO is one of the varieties of Arduino board microcontroller based on ATmega 328P, which takes input from the sensors and is connected to the Wi-Fi with the help of the Wi-Fi module [7-8].

\section{Conclusion}

The personal weather report design developed in this project has the power to simplify everything within your home and life. As people are getting used to the easy use of smart phones and other devices which can access the internet we can easily introduce it practically. For instance in the case of a potential gas leak, your single best detector is your own nose. You can supplement your power of smell with a gas sensor, which will test for i-butane, hydrogen, methane, etc. Gas leaks are rare, but they can set off catastrophic explosions, so it will ensure the monitoring of such incidents. It can be further extended as a wireless climate control device for your surroundings. Maintain ideal temperature, prevent frozen pipes and protect your pool and many other such applications. It can also be used to develop a home temperature monitoring system to ensure the comfort and safety of your family, and it protects your home and valuables from serious damage. Digital temperature sensors, which are combined with humidity sensors in this project can be placed in the areas of your home where you want to measure and monitor temperature. Further if it is set up to connect to a home security monitoring system. Whereas the possible applications of the pressure sensor could be automatic door bell, carpet alarm, hit switch, knock bell, garage alarm, door mat sensor. The major advantage of this project is that one can view/ or take care from anywhere under the Wi-Fi access, this project can find various applications in a number of fields which can be certainly used for the benefit of society and mankind as a whole in this fast pacing world.

\section{Design}

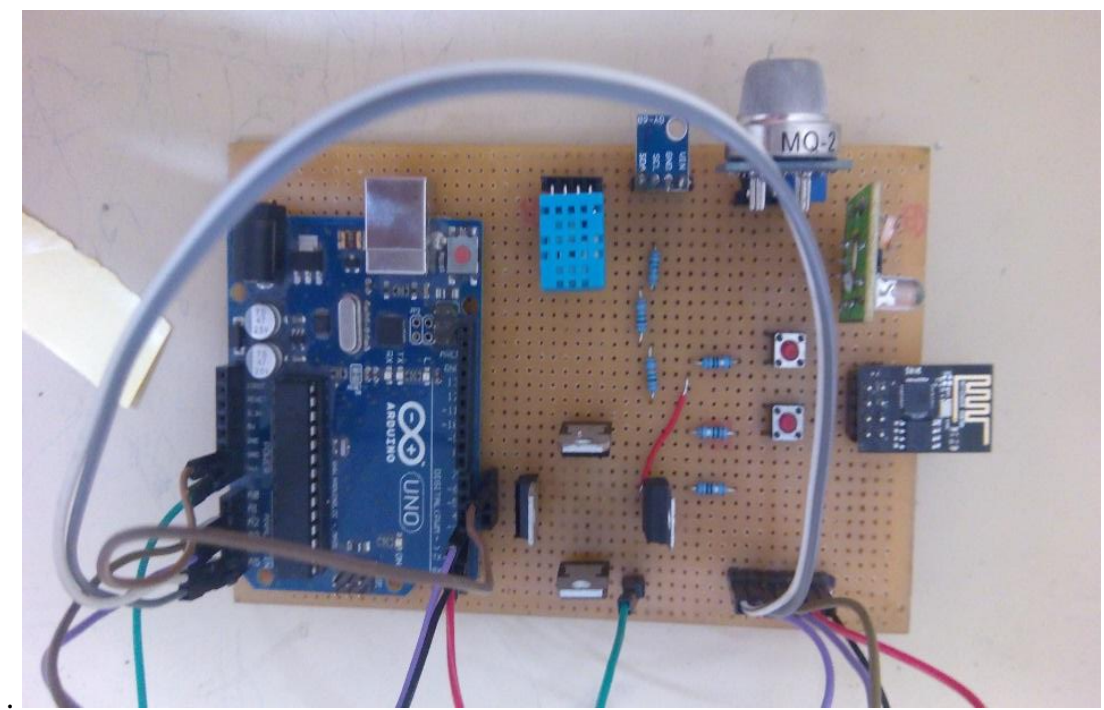

Figure 1. Model of Project

\section{References}

[1]. Brown, Eric (13 September 2016). "Who Needs the Internet of Things?". Linux.com. Retrieved 23 October 2016.

[2]. Brown, Eric (20 September 2016). "21 Open Source Projects for IoT". Linux.com. Retrieved 23 October 2016.

[3]. "Internet of Things Global Standards Initiative". ITU. Retrieved 26 June 2015.

[4]. "Internet of Things: Science Fiction or Business Fact?" (PDF). Harvard Business Review. November 2014. Retrieved 23 October 2016.

[5]. Evans, Dave. "The Internet of Things: How the Next Evolution of the Internet Is Changing Everything" (PDF). (April 2011) Cisco. Retrieved 15 February 2016.

[6]. Hendricks, Drew. "The Trouble with the Internet of Things". London Data store. Greater London Authority. Retrieved 10 August 2015.

[7]. "Arduino", arduino.cc, 2010. [Online]. Available: http://www.arduino.org/products/boards/arduino-uno. [Accessed: 10-sep-2016].

[8]. "Wikipedia", Wikipedia.com, 2010. [Online]. Available: https://en.wikipedia.org/wiki/Arduino. [Accessed: 10-sep-2016].

[9]. Brian Benchoff (August 26, 2014). "New Chip Alert: The ESP8266 Wi-Fi Module)", Retrieved 2015-06-24. 


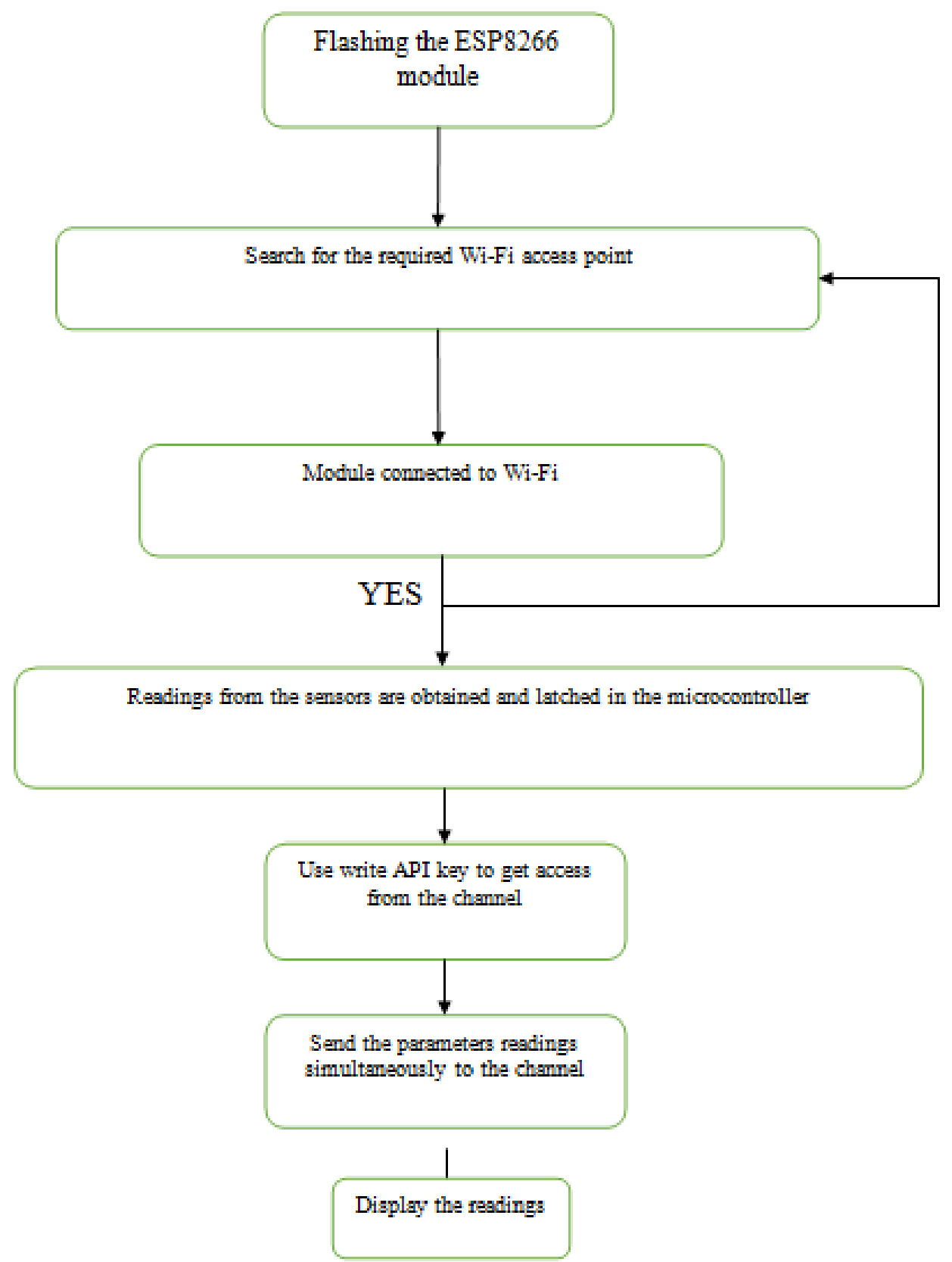

Figure 2. Flowchart 


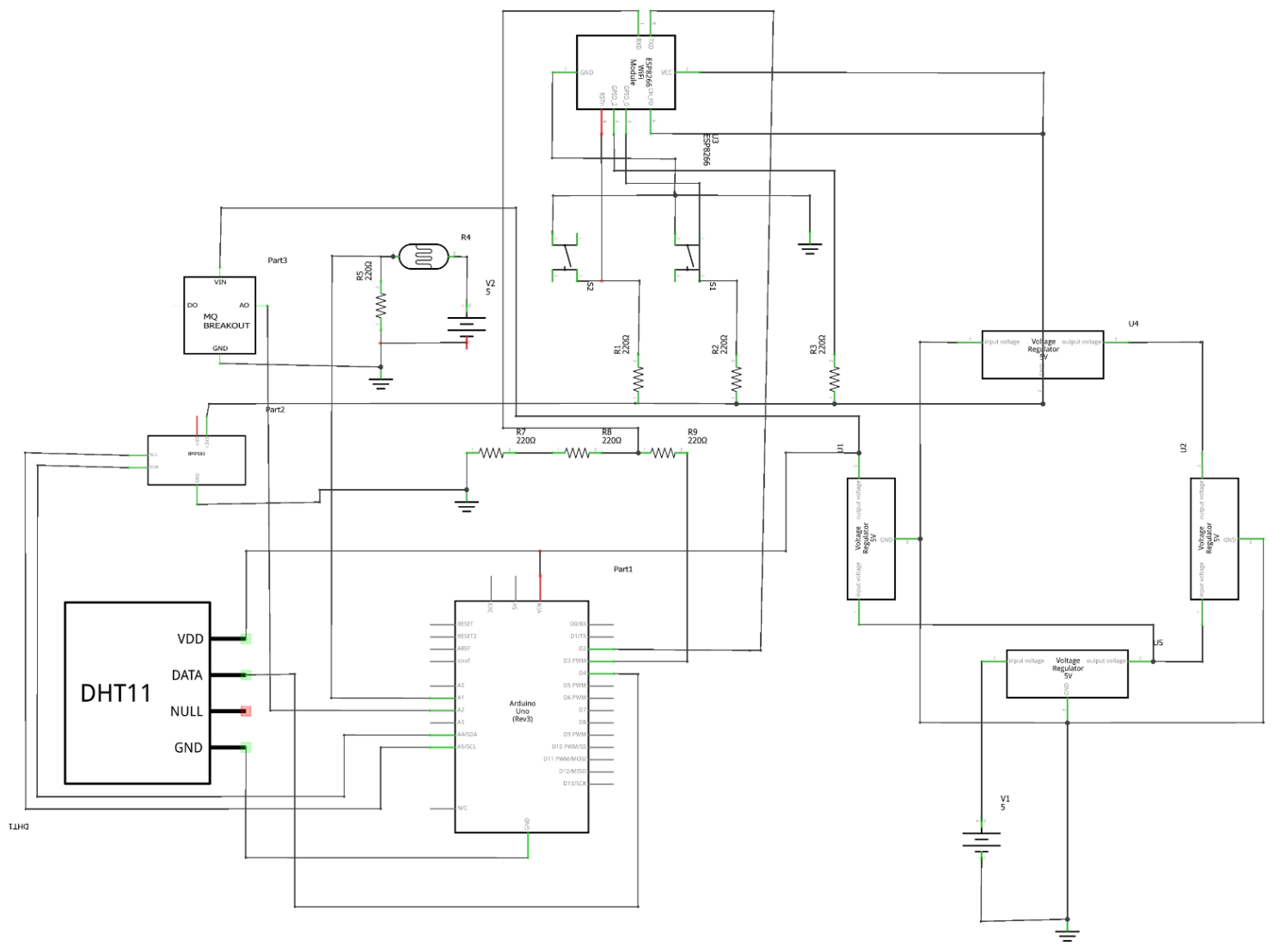

Figure 3. Circuit Diagram 